\title{
Wireless technologies for the Construction Sector Requirements, Energy and Cost Efficiencies
}

\section{Heller, Alfred; Orthmann, Christian}

\section{Published in:}

Energy and Buildings

Link to article, DOI:

10.1016/j.enbuild.2013.12.019

Publication date:

2014

\section{Document Version}

Early version, also known as pre-print

Link back to DTU Orbit

Citation (APA):

Heller, A., \& Orthmann, C. (2014). Wireless technologies for the Construction Sector: Requirements, Energy and Cost Efficiencies. Energy and Buildings, 73, 212-216. https://doi.org/10.1016/j.enbuild.2013.12.019

\section{General rights}

Copyright and moral rights for the publications made accessible in the public portal are retained by the authors and/or other copyright owners and it is a condition of accessing publications that users recognise and abide by the legal requirements associated with these rights.

- Users may download and print one copy of any publication from the public portal for the purpose of private study or research.

- You may not further distribute the material or use it for any profit-making activity or commercial gain

- You may freely distribute the URL identifying the publication in the public portal 


\section{Accepted Manuscript}

Title: Wireless technologies for the Construction

Sector-Requirements, Energy and Cost Efficiencies

Author: Alfred Heller Christian Orthmann

PII:

S0378-7788(13)00834-7

DOI:

http://dx.doi.org/doi:10.1016/j.enbuild.2013.12.019

Reference:

ENB 4709

To appear in: $\quad E N B$

Received date: $\quad$ 5-6-2013

Revised date: $\quad$ 16-10-2013

Accepted date: $\quad$ 12-12-2013

Please cite this article as: A. Heller, C. Orthmann, Wireless technologies for the Construction SectorndashRequirements, Energy and Cost Efficiencies, Energy and Buildings (2013), http://dx.doi.org/10.1016/j.enbuild.2013.12.019

This is a PDF file of an unedited manuscript that has been accepted for publication. As a service to our customers we are providing this early version of the manuscript. The manuscript will undergo copyediting, typesetting, and review of the resulting proof before it is published in its final form. Please note that during the production process errors may be discovered which could affect the content, and all legal disclaimers that apply to the journal pertain. 


\section{Wireless technologies for the Construction Sector - Requirements, Energy and Cost Efficiencies}

Alfred Heller, Christian Orthmann

Department of Civil Engineering, Building 118, Brovej, DTU, Technical University of Denmark, +454525

1861, alfh@byg.dtu.dk

Abstract - The construction sector has been rather reluctant with respect to the implementation of ITC innovations that other industries have adopted for years. One of the reasons could be the lack of services by the proposed innovations especially the RFID solutions. This technology is well-researched within the building sector and is therefore used to analyse requirements for alternative technologies. The motivation of the current work is to find upcoming technologies that bring improvements into the sector, for example improved life cycle costs and energy efficiencies, increasing quality, construction and operation efficiency and reducing faults and losses.

The paper also lays out requirements expected by the sector. It will be shown that the wireless sensor network technology is a strong competitor that may meet the requirements. By analysing the application of such technologies throughout the building lifecycle, the utilization can be manifold, hereby minimising overall economic costs and maximising the added values for all involved stakeholders.

Based on the expectations of the sector, the experiences with the introduction of the RFID technology and by estimating the applicability of the extra services that follow the wireless sensor network, the paper will line up the requirements that the new technology has to meet to be introduced successfully.

Keywords: Wireless Sensor Networks; RFID; Building Sector; building lifecycle; requirements, cost, energy efficiency 


\section{Introduction}

The basic idea behind the current work is that there is a need for innovation within the construction and building sector due to, among others, too high margin of errors and necessity of cost reduction. The economic impact of technology can be significant. A Danish consultant report estimated that the savings resulting from error avoidance can reach up to $13-18 \%$ of the construction sum [1]. Reduction of losses due to theft, more efficient communication and documentation methods and better construction site management all provide additional economic efficiency.

One of these innovations has been to introduce Radio Frequency Identification (RFID). The technology made no breakthrough within the construction sector as it did in other sectors and industries. The current paper gives some ideas, why this happened. From the experiences with RFID, specifications can be drawn for a new attempt to introduce alternative technologies that are more readily accepted by the construction sector and lead to the desired increase in efficiency, quality, and consequently economical improvements.

The paper proposes wireless sensor networks (WSN) as a promising technology that will bring along a number of additional values. To support the proposal, the value creating services demanded by the sector are analysed and transformed into requirements that the upcoming technology has to meet and that can be tested in further work.

\subsection{Motivation}

The main motivation of the current work is to reduce the environmental impact of energy usage of buildings. It is our proposal that the application of sensors play a vital role in this respect, enabling insight into the operation and control of the building energy systems. Further arguments for the introduction of wireless sensoring to building processes are:

- Improving efficiency in the building process.

- Improving operational efficiencies including energy performance during operation. 
- Economical efficiencies and savings.

With respect to energy efficiencies of buildings, work by the Danish Technological Institute shows potential energy savings between $10-30 \%$ by adjusting the different sensors and control settings [2]. Others demonstrate savings based on a commissioning process of between 7-12\% [3] and [4], referes to a number of other published work on energy savings due to commissioning. More support are to be found in [5] and [6] which propose energy savings in less quantified terms. The argument of the current paper is that increased flexibility in monitoring systems will entail similar savings as found in literature, but even improve these potentials through e.g. increasing insight, the ability to "debug" the systems and such like. While commissioning is often a onetime activity the above energy savings can be lost due to many reasons, e.g. changes in installations, demands and repurposing of the buildings. Continuous Commissioning is proposed to address these issues and obtain the energy savings for the life span of the building. Flexible sensoring will increase this approach.

Building sensoring is not new. In fact most buildings today are subject to some form of sensoring. Examples of existing building sensoring applications could be: Central or Building Monitoring Systems for large buildings, home surveillance and automation, monitoring and control for small buildings. Today, building sensoring is commonly wired; meaning that the sensors communicate through wiring established in the building. The introduction of wireless sensors brings a number of economic advantages:

1. Expenses for wiring are reduced.

2. Expenses for planning of wiring is eliminated or reduced dramatically.

3. Sensors can be retrofitted even in existing buildings.

Going one step further in the technologies of wireless sensoring, WSN provide even more advantages to the building sector: 
1. Flexibility of adding or removing sensors without interruptions or demands for reconfiguration.

2. Inspired by the IT development processes, the application of sensors can bring a new level of monitoring and control into the building, based on known IT skills, tools and procedures.

a. Testing on basis of standard environments. Running test sequences that can be "knowledge based", e.g. based on Al, simulations and other advanced methods.

b. Agility/flexibility in hardware and services.

c. Interface flexibility that is achieved through web-based and other interfaces.

d. Device flexibility (PC, smart phones and other devices).

e. Backbone standardizations, flexibility and low cost.

Below, we take a look at the wireless sensor network technology after an historical view on technologies involved.

\subsection{From identification towards sensoring - A historical view}

The identification of artefacts plays a central role in any process. If you want to control something, you have to be able to identify it. Technologically, this has been addressed firstly by simple labelling that evolved to "tags", and more recently QR-barcodes. To enable identification and at the same time the storage of a minimal set of information, new electronic solutions are developed and we can expect this evolution to continue in the future.

The aim of this section is not to give a comprehensive survey on the history of identification technologies, but rather to show the main aspects of the different steps in the development of identification schemas. Not surprising, some kind of "label" was probably the first kind of identifier of things - You may have seen your grandmothers jam labelled with some stickers with a hand written "identification" - "Strawberry Jam, $1932 "$. 
Labels where the only available technology before the IT-revolution and is still widely used. The strength of the technology is its simplicity and low price, whereas the weakness lies in the many things that they cannot provide. Labels must have front-to-front contact with the reader which is a rather pivotal limitation for automatic applications. There are also limitations on the information that can be provided through labels. Some of these shortcomings are resolved in the following technologies.

Radio Frequency Identification (RFID) was already available in 1945 as an espionage device by the Soviet Union for identification. Parallel to this development, transponder-based solutions popped up and a more advanced RFID was presented by Mario Cardullo in 1973. The Cardullo [2] technology was introducing as a transponder with memory, hence being able to relate the identification with extra meta-data/information read by external readers. Michael [3] presents a history of automatic identification technologies, from bar codes, to magnetic-stripe cards, smart cards, biometrics and RFID tags, with a very large literature survey on the topic. A reasonable set of applications throughout history are stated by Russos and Vassilis [4].

RFIDs are small electronic devices that can be incorporated in almost any kind of object, clothes, devices, food wrapping, and can be applied almost everywhere. The information is accessed through a reader with no contact, overcoming the limitation of the label's need for front-to-front contact between the "tag" and the reader, and enables automation of this process, reducing labour requirements. The technology is designed in three basic types, "passive" not using any batteries and activated by the readers, "active" that use batteries to actively communicate with readers, and a third that combines the two by activation of passive devices to an active mode. In any case, the strength of the technology is its simplicity and low price, whereas the drawback of the technology lays in the limited memory capacity. However, at an early stage the strength of the Internet was coupled to overcome this limitation - connecting the identifier to an internet address provides new opportunities for information storage and new business models of pooling of information, as we will see below. 
In addition to RFID, there are various kinds of wireless technologies including mobile phone solutions and communication solutions such as WIFI, both are capable of communicating over large distances. On the other end of the spectrum there are "near field communication" solutions.

To establish evidence for the relevancy of the introduction of the alternative technology, the well-known and well-researched RFID-technology is analysed for their proposed impacts and lack of capability reported in literature. Using RFID-technology as a basis, more advanced wireless sensor network technology is examined for further testing of the hypothesis. Identification is one of the main features of both mentioned technologies, but both lack globally uniqueness.

In the current paper an alternative approach "Wireless Sensor Networks" will be proposed, solving the limitations of the RFID solution and providing additional benefits. Before doing so, the application of RFID within building construction and building management is examined to give an idea of the potentials for such technologies and also give us specifications for the functionalities that ought to be met by an alternative solution.

\section{RFID in construction and building sector}

To establish evidence for the relevancy of the introduction of the alternative technology, the well-known and well-researched RFID-technology is analysed for their proposed impacts and lack of capability reported in literature. Using RFID-technology as a basis, more advanced wireless sensor network technology is examined for further testing of the hypothesis.

In 2006 the application of RFID within the building sector or building management was rather limited even though billions of devices where used in the food industry, warehousing, industrial production, and especially supply chain management involving different actors [4]. A report by ERAbuild [5] gives a comprehensive overview of the subject and reports on applications and research work. The conclusion was 
that the achievements from other sectors and industries ought to be exploited in the building sector, such as:

1. direct and automated surveillance maintenance programmes by a click on a PC or PDA

\section{2. inventory control}

3. control of right equipment at right place

4. reduction of data entry errors both during 'production' and afterwards in maintenance

However, the report concludes that the implementation in the building industry had not been seen in 2006, even though vendors from industries did act as drivers for the development. A rather important finding was reported by The Danish Building Research Institute [6]:

In the construction value chain from manufacturer of building materials, distributors, constructors, operators and end users many advantages can be achieved from embedded technology (RFID). However there are very different demands and needs to performance of technology in the different groups.

The main application for RFID is reported to be related to facility and asset management, but also access control to building sites. These applications tend to give pay backs in form of immediate savings. Cases are reported where construction details, drawings and instruction videos on the web are linked through a RFID and related "tag" information. The application of Building Information Models (BIM) in relation to RFID tags are reported, enabling real time tracking of construction progress, similar to a supply chain monitoring.

As we will find below, the current research takes this diversity into consideration. The report by [5] finally concludes:

At this stage in use and development of RFID in Construction we must realise that there are many questions and few answers, most cases that we have been investigating are pilots and not full-scale implementations,

\footnotetext{
${ }^{1}$ Note that the device technologies have changed in time.
} 
many research papers and development projects have caused great interest and many observers but not any steps into fulfilment of RFID strategies.

Since, the application of RFID is still on-going: Chang, Hung and Peng [7] proposes the application of RFID in combination with sensors cast into wall constructions, and many other applications for crack monitoring are reported. Jacobsen and Poulsen [8] propose the introduction of RFID for quality control and [9] proposes the application of RFID for material identification. Such efforts are sporadic and no master plan seems to lead the development. In the meantime other technologies are pushing, amongst them the Home Entertainment and Surveillances segment, applying wireless Zigbee, Z-wave and other wireless communication technologies. Building health monitoring applications are also presented in research, [10] and [11], applying the below promoted sensor network technology.

By analysing the literature of the involved technologies a number of findings can be extracted. Chang et all.

[12] Table 3 and 4, show clearly that the RFID technology was published widely but with low "depth of investigation" in many sectors and industries, the construction industry accounts for 4 out of 316 published papers. These findings are also supported by [1].

\subsection{Economic considerations based on construction lifecycle costs}

The building lifecycle is e.g. defined in [13] to consist of the following phases: pre-design > design > construction $>$ operation $>$ refurbishment $>$ decommissioning $>$ deconstruction and demolition. By simplifying to relevant tasks for the current study and adding commissioning to the lifecycle, due to the fact that quality assurance and energy efficiency is documented and established at this stage, we use the following lifecycle in the current work:

construction $>$ commissioning $>$ operation $>$ refurbishment $>$ decommissioning $>$ demolition Regarding commissioning: There are numerous reports on flaws and errors that could be handled during this phase that would lead to a long-term increase in efficiencies. The current system proposal supports 
commissioning especially well due to its flexibility, where sensors can be added or removed dynamically without disturbing the operation of the network and with no configuration necessary. This is one of the most powerful features of this technology.

The hypothesis of this work is that - The introduction of state-of-the-art identification, sensor and communication technologies to the building lifecycle leads to significant economical, technical and societal gains. If sensors are wireless, especially in form of wireless sensor networks, these gains are even more pronounced.

One of the main arguments for the current solution is that by repurposing the id and sensor components during the lifecycle of buildings, the economic costs become insignificant and the values due to increased services result in efficiency improvements on many performance parameters of the building. The current paper investigates the application of wireless sensor technologies in the given lifecycle-phases and documents the potential of repurposing of the same sensors for different conditions throughout the lifecycle.

\section{Wireless Sensor Networks for the Construction Industries}

It is apparent from the above status on the application of RFID that there are significant technological and logistical improvements that have not been utilized within the building sector. Hence a more advanced technology is required. In the current paper a wireless network technology is proposed that adds additional services to the identification devices. Due to the fact that the technology is combining many subtechnologies, see Figure 1, it will be presented in detail here.

WSN provides identification of the sensors by wireless communication and storage of information in its memory. This is very similar to RFID. The advance comes from the combination with sensors that add information from the surroundings, and network communication capabilities. The implication of these will 
be explained below. In the following a brief introduction to the terminology used in this report is presented.

A WSN consists of a variety of configurations of distributed "nodes". The individual nodes can be of different types:

Synchronization node: A node which collects data from other nodes in the network and possibly sends the synchronized data to a PC or server. Synchronization nodes are in some cases called Terminals or Gateways.

Node / Sensor node: A device collecting data through sensors and possibly sends collected data to synchronization nodes. Sensors typically monitor environmental or physical conditions like humidity and temperature.

Nodes are capable of communicating in "hops"; meaning that they communicate with devices in the network through each other. This means that any two nodes in a wireless sensor network can communicate by having messages or data passed through other nodes. Effectively, nodes don't physically need to be within the communication range of their targets to communicate.

\subsection{Functionalities required derived from the building lifecycle application}

From the above investigation on RFID and the analysis of the demands of the building cycle, we propose the following utilization of the wireless technology for the building lifecycle:

- Production (quality assurance, documentation, facility management)

- Transport (event registration)

- Construction (identification, facility management, assembly support)

- Commissioning (documentation, adjustments)

- Operation (monitoring, optimization) 
- Disposal (identification, metadata, waste management)

Services that a future technology must provide that correspond with the proposed utilization:

- Documentation of building components (identification, information storage)

- Easy communication of id and information with existing technologies

- Remote access

- Online warnings - testing

- Flexibility (add and remove sensors in running system with no reconfiguration)

The added values of such a solution are:

- Reduction of errors during design, construction, commissioning, operation.

- Better building performance due to better monitoring (energy, indoor environment).

- Better facility management services due to increased information.

- Cheap and standardized technologies (outside the network itself).

From the collected functionalities the following requirements can be derived for the communication technologies that are to be investigated":

Basic functionalities: These are "identification" of the sensor, memory for minimal information storage over time, which are very similar to the RFID technology. Due to the fact that WSN do store sensor data, the memory space is much larger than for RFID solving this limitation.

Wireless communication: RFID stays passive in regard to communication, while WSN broadcast according to the requirements of the given application. The interval of broadcasts, the amount of information 
communicated and the strength of the antenna, all contribute to the characteristics of the actual applications. It also has a large impact on the battery lifetime, discussed separately.

RFID can only communicate to special devices (base stations), while the nodes of WSN can communicate with each other, called "communicating in hops". This introduces much wider application of this technology.

Due to the fact that synchronisation nodes do not have to be very advanced, it is possible to use many in the same installation adding another set of flexibility into the overall solution, e.g. changing from special protocols and tools to well-established IP protocol that enables the utilization of the many tools that are compatible with the internet protocol. This again gives a large space of freedom in system design and economical savings, because no special hardware is used for handling the collected data and information.

Positioning - localisation: This functionality is not really a demand by the sector, but is an extra service by WSN that provides many possibilities for localisation of material, components, people and others.

Long lifetime of battery: The weakness of WSN is the battery lifetime. There are a few strategies to overcoming this obstacle:

1. Saving energy demand is the most applied solution. This is achieved by optimizing all energy related parameters of the nodes, leading to the wide range of different WSN solutions instead of standardisation. An important requirement here is the demand for state changes where the node changes its behaviour, e.g. frequency of sensor readings and broadcasts, to limit energy demands.

2. Increasing the battery volume which is expensive and requires more space.

3. Introducing energy harvesting in any means. This is an on-going research and development area.

4. Wiring for energy supply, destroying the wireless concept. On the other hand, the wiring can be cheap, simple and short distance. 
5. Wireless energy supply, which also is under development and cannot be implemented as a commodity solution.

\subsection{Discussion and further work}

The paper analyses the lack of break-through for the RFID technology within the construction sector, despite the demand for innovation to reduce the number of errors and improve overall construction efficiency. By analysing experiences from the technology a number of demands were found, which could be transformed into requirements for an alternative technology that may have the chance for widespread implementation in the sector and hereby lead to the expected gains. In future work the requirements can be tested on WSN and other technologies.

A common concern in a WSN is the necessity of batteries in general and especially the battery lifetime. The issue is discussed in literature and several sources mention battery life times in WSN of up to 30 years; even when sending data every minute. [14] [15]. Most sources like [16] only guarantee battery life of about 5 years.

Another untouched question is what limits there are on the range of nodes in a WSN. This is dominated by the antenna characteristics and should be studied very carefully. There exist both long- and short-range WSNs, thus making it possible to fit the network to the application needs. In [17] a long range WSN was tested with a node range of $13.2 \mathrm{~km}$, however the nodes were of significantly larger size than the Sun SPOTs used in the project-experiments. Examples of nodes with a more realistic size for the intended applications are described in [18] these nodes had a range of about $300 \mathrm{~m}$. In regard to the building process $300 \mathrm{~m}$ is more than enough for most cases and it could be argued that a smaller range would do as well due to WSNs' ability to communicate in hops. Node range has a dramatic impact on battery lifetime.

The interaction of building information models (BIM) with identification technologies such as RFID and WSN will also be relevant to investigate. 
The current study indicates that it is technically feasible to utilize wireless sensors in a network throughout the building process. That said, several important issues were identified and solutions should be considered carefully in preparation for further development.

Introducing sensors with identification capacities at similar costs enhances the services during the lifecycle of building design and construction, enabling the sensors to adjust to the relevant mode of operation. This way the cost of these extra sensors will be funded in the earliest stage of the lifecycle, while still generating savings during the remaining lifecycle stages.

One open question is the possibility for integration of the wireless sensor network with Building Monitoring Systems (BMS) and CMS. The proposed internet connected WSN has its own "backbone" infrastructure (server, database and similar). Hence questions arise on how to integrate them, if the backbone is redundant, or opposite the backbone is an important support and gateway for the BMS. Questions also arise on how to integrate with other wireless services such as Home Automation and more. This is left to later research.

The ability of localisation of the WSN technology increases the service pallet even further, enabling reasonably exact localisation of the sensors themselves and hereby moving objects and even humans, thus enabling emergency situation management and much more.

This vision has already been demonstrated in various implementations. A Danish consultant company, BMT Instruments, provides a moisture monitoring service for clients that have access through the internet to their data. Many others examples can be found. The limitation of these implementations is that they are proprietary, which blocks changes to the behaviour of the sensors, the network and its communication. Therefore research with such systems is very limited in their purpose of designing next generation wireless solutions for the construction sector. Therefore future work will be to develop an open platform implementation that overcomes these barriers. The idea is to design the hardware on open components and build the software infrastructure based on open protocols. The only exception to that is the 
communication between sensors and the terminals that may depend on some proprietary protocol, whereas the rest is handled within the web technologies.

Another future development that would implement actuation and decision automation into the overall technology, is described for "Smart Objects", [19], where both RFID and WSN would play a role. Here, more intelligence is placed into the sensors; even going so far as to utilize "embedded systems" into the individual sensor units.

\section{Bibliography}

[1] Byggestyrelsen, »Report on flaws and shortcommings in construction (in Danish),« 2010. [Online]. Available: http://www.ebst.dk/file/103339/rap_fejlogmangler.pdf.

[2] P. P., EiSE - Energy optimization method (Danish), Taastrup, Denmark, 2013.

[3] L. Wang, G. S., J. Fiegel og et.al., "Monitoring-based HVAC commissioning of an existing office building, « Applied Energy, årg. 102, pp. 1382-1390, 2012.

[4] M. C. M., »Existing-Building Commissioning, « HPAC Engineering, årg. 85, nr. 8, p. 18, 2013.

[5] S. Ginestet, D. Marchio og O. Morisot, »/mprovement of buildings energy efficiency: Comparison, operability and results of commissioning tools, « Energy Conversion and Management, årg. 76, p. 368376, 2013.

[6] H. Grindvoll, O. Vermesan, T. Crosbie og et.al., "A wireless sensor network for intelligent building energy management based on multi communication standards - A case study, « Journal of Information 
Technology in Construktion, 2012.

[7] G. Roussos og K. Vassilis, »RFID in Pervasive Computing: State-of-the-art and Outlook., Pervasive and Mobile Computing, årg. 5, nr. 1, pp. 110-131, 2009.

[8] (en), Wikipedia, »RFID, « [Online]. Available: http://en.wikipedia.org/wiki/Rfid.

[9] K. Michael, "Trends in the Selection of Automatic Identification Technology in Electronic Commerce Application, " i Trends in the Selection of Automatic Identification Technology in Electronic Commerce Application, Talca, Chile, N. Cerpa \& P. Bro (eds.), 2003.

[10] The Danish Building Research Institute, »Embedded technologies in Construction, 2006.

[11] ERAbuild, »Review of the current state of Radio Frequency Identification (RFID) Technology, its use and potential future use in Construction, 2006.

[12] SPIE(a), »Smart Sensor Phenomena, Technology, Networks, and Systems Integration, 2012.

[13] SPIE(b), "Sensor and Smart Structures Technologies for Civil, Mechanical, and Aerospace Systems," 2012.

[14] C. S. Birch, »Ph.D. Thesis: Virtual Model Linked with Physical Components in Construction, "Aalborg University, 2009.

[15] K. S. Birch, P. Christiansson og K. Svidt, »Ontologies to Support RFID-Based Link between Virtual Models and Construction Components, "Computer-Aided Civil and Infrastructure Engineering, årg. 25, nr. 4, p. 285-302, 2010.

[16] T. Berner-Lee, J. Hendler og O. Lassia, „The Semantic Web - A new form of Web content that is

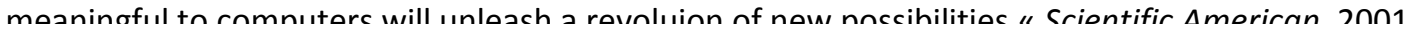


meaningful to computers will unleash a revoluion of new possibilities, "Scientific American, 2001.

[17] »EPCglobal standards overview, « 2008. [Online]. Available: http://epcglobalinc.org/standards.

[18] J. Brase, A. Farquhard, A. Heller og et al., „Approach for a joint global registration agency for research data, "Information Services and Use, årg. 29, nr. 1, pp. 13-27, 2009.

[19] D. Jacobsen og L. Poulsen, »Mr. thesis: Innovation in the building sector - Implementation of RFID in quality control (in Danish), « Aalborg University, 2011.

[20] T. S. López, C. D. Ranasinghe, B. Patkai og D. McFarlane, »Taxonomy, technology and applications of smart objects, « Information Systems Frontiers, årg. 13, nr. 2, pp. 281-300, 2011.

[21] M. Georgiadou, T. Hacking og P. Guthrie, »A conceptual framework for future-proofing the energy performance of buildings, « Energy Policy, årg. 47, p. 145-155, 2012.

[22] C. Orthmann, B.Sc. Thesis: Positioning and registration of items $n$ a mobile context., Technical University of Denmark, 2011.

[23] M. Thomassen og D. V. Lebech, B.Sci. Thesis: Anvendelse af sun spots til sensorvetværk. (Danish for Application of sun sport sensor networks), Technical University of Denmark, 2008.

[24] J. Xu, L. Wei og et.al., »Distance measurement model based on rssi in wsn, « Wireless Sensor Network (WSN), årg. 2, nr. 8, pp. 606-611, 2010.

[25] A. M. Rais, R. S. Kumar og et.al., "Statistical analysis of wsn based indoor positioning localization schemes with kalman filtering., "International Journal of Electronics Signals and Systems, årg. 1, nr. 3, pp. 36-41, 2012. 
[26] Monnit Corp.;, "Monnit industrial accelerometer.," 2013. [Online]. Available:

http://www.monnit.com/products/industrial-wireless-sensors/accelerometer.php.

[27] National Instruments Corporaton, »NI wireless reference to the product description., " 2013. [Online]. Available: http://sine.ni.com/np/app/main/p/ap/imc/lang/da/pg/.

[28] M. Saxena, P. Gupta og B. Jain, »Experimental analysis of rssi-based location estimation in wireless senor networks, « i 3. International Conference on Communication Systems Software and Middleware and Worshops, 2008.

[29] X. Lou, W. O'Brien og C. L. Lulien, „Comparative evaluation of received signal-stength indes (rssi) based indoor localization techniques for construcation jobsides., "Advanced Engineering Informatics, årg. 25, nr. 2, pp. 355-363, 2011.

[30] S. Wang og H. Hu, „Wireless sensor networks for underwater localization: A survey.»" Technical report, University of Essex, 2012.

[31] C. Boano, J. Brown, T. Voight, J. Brown og U. Roedig, "The impact of temperature on outdoor eindustrial wsn applications., " IEEE Tansactions on Industrial Infomatics, årg. 6, nr. 3, pp. 451-459, 2010.

[32] Powercast Corporation;, »The lifetime power wireless sensor system., 2013. [Online]. Available: http://www.powercastco.com/products/wireless-senor-system/..

[33] T. Dittrich, H. Manachem og et.al., "Lithium batteries for wireless sensor networks. Technical report.," Tadiran Batteries, 2010.

[34] S. Willis og J. Kikkert, "Radio propagation model for long-range wireless sensor networks, " i 6th

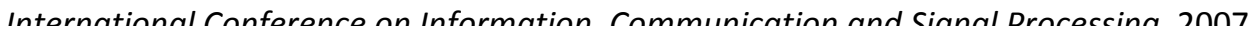


International Conference on Information, Communication and Signal Processing, 2007.

[35] Oracel Corporation, „Sun spot - General reference to the Sun SPOT World Forums, « 2013. [Online]. Available: http://www.sunspotworld.com/forums.

[36] Wikipedia Foundation Inc., »General reference to the Sun SPOT Wikipedia page, « 2013. [Online]. Available: http://en.wikipedia.org/wiki/Sun_SPOT.

[37] Corporation for National Research Initiatives (CNRI), »The Handle System, " [Online]. Available: http://www.handle.net/. [Senest hentet eller vist den 2013].

[38] C.-C. Chao, J.-M. Yang og W.-Y. Jen, »Determining technology trends and forecasts of RFID by a historical review and bibliometric analysis from 1991 to 2005, « Technovation, årg. 27, nr. 5, p. 268279, 2007.

[39] C.-Y. Chang, S.-S. Hung og Y.-F. Peng, "An evaluation of the embedment of a Radio Frequency Integrated Circuit with a temperature detector in building envelopes for energy conservation, « Energy and Buildings, årg. 43, pp. 2900-2907, 2011.

[40] E. Esgen og B. Akinci, »An Overview of Approaches for Utilizing RFID in Construction Industry, « i PROCEEDINGS OF THE 1ST RFID EURASIA CONFERENCE, 2007. 
Table of Figures:

Figure 1 Functionalities of wireless sensor networks. 

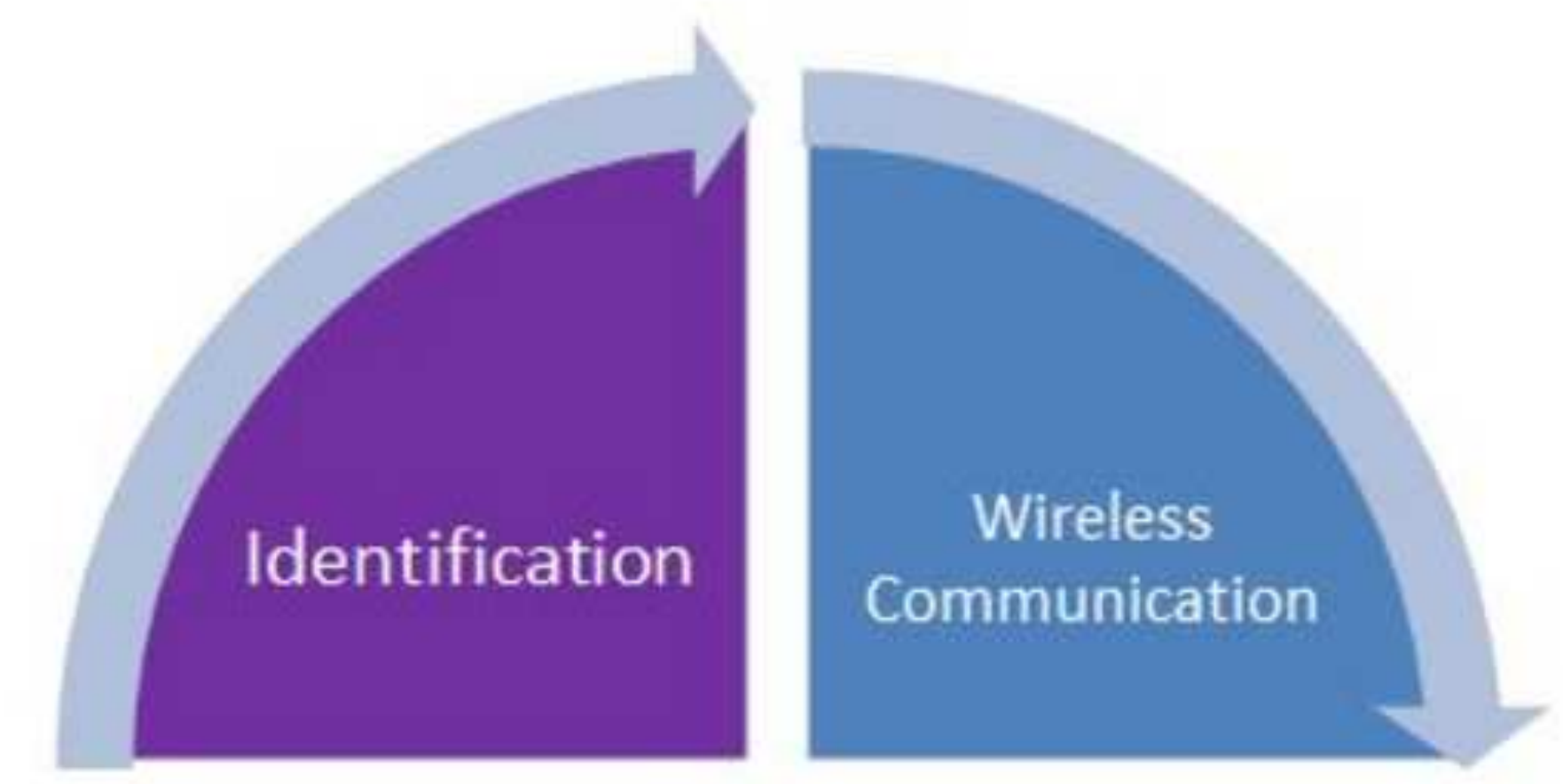

Memory

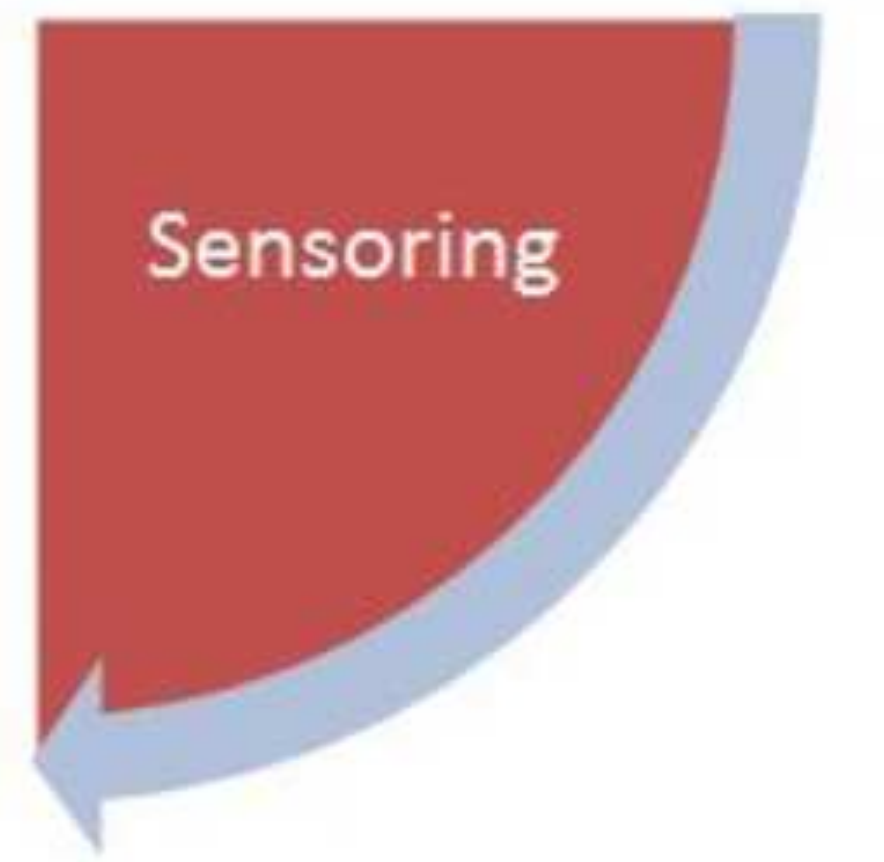

rage 21 or 21

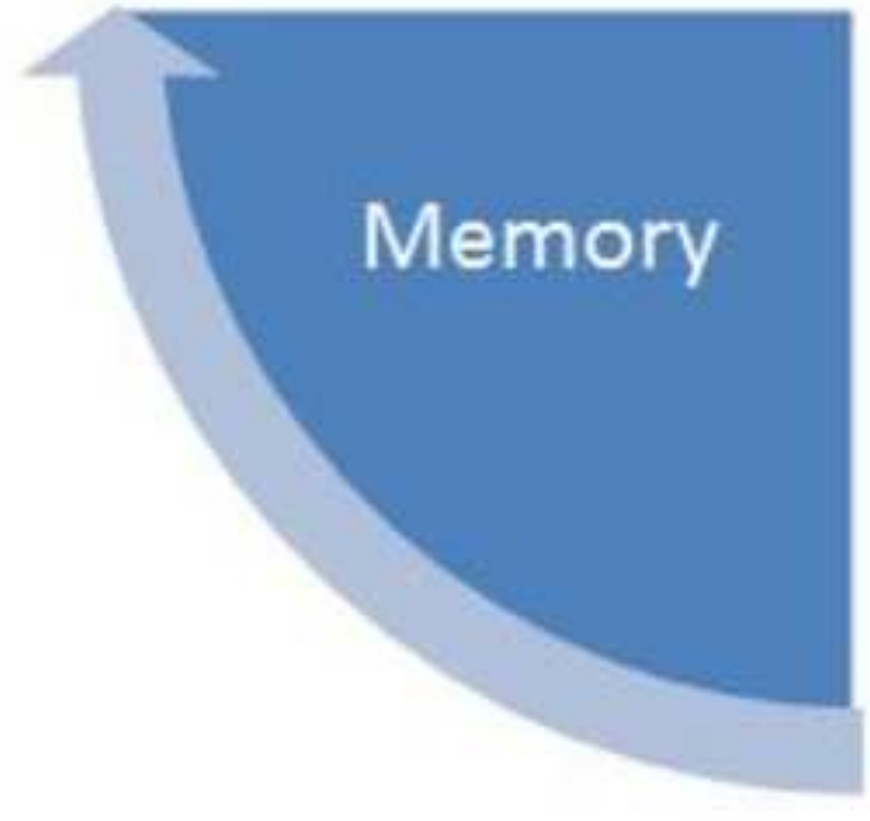

